\title{
On the Role of Self-similarity in the Dynamics of Effective Field Theory
}

\author{
Ervin Goldfain \\ Research Scholar, Ronin Institute, Montclair, New Jersey 07043 \\ Email: ervin.goldfain@ronininstitute.org
}

\begin{abstract}
The goal of this work is to show that the underlying symmetries of effective field theory can be traced to the onset of self-similarity. In particular, we argue that the scale-free structure of fractal geometry lies at the heart of invariance principles in classical and Quantum Field Theory.
\end{abstract}

Key words: Self-similarity, fractal geometry, generalized homogeneous functions, general covariance, self -organized criticality, Lie groups, effective field theory.

\section{Introduction}

The relevance of continuous symmetries in theoretical physics can hardly be overstated. Conservation laws form the backbone of both quantum and relativistic phenomenology and are organically tied to Lorentz symmetry, gauge groups and the diffeomorphism invariance of General Relativity.

A set of observables $q=\left\{q_{1}, q_{2}, \ldots, q_{N}\right\}$ associated with a system may be naturally represented as coordinates of an $N$-dimensional vector $\mathbf{q}$ in $q$-space. The general principle of invariance demands that the laws of physics must be independent of any origin or orientation of $\mathbf{q}$. Moreover, the general principle of covariance demands form 
independence of the laws of physics from any particular choice of coordinates in $q-$ space.

The goal of this brief report is to show that fundamental symmetries of effective field theories emerge from self-similarity, the latter being inspired by the geometry of fractal and multifractal sets. As we shall see below, in the context of theoretical physics, selfsimilarity reflects invariance of the action under the most general scale transformation related to dilations, rotations, and translations [Appendix A, 1, 14].

\section{Noether theorem from scale transformations}

Consider a classical field theory with fields $\varphi_{i}$ and action

$$
S=\int d^{4} x L\left(\varphi, \partial_{\mu} \varphi\right)
$$

An infinitesimal transformation of coordinates and fields can be formulated in terms of a set of $l=1,2,3, \ldots, N$ infinitesimal parameters $\varepsilon^{l}$ as in [2]

$$
\begin{gathered}
x^{\mu} \rightarrow x^{\prime \mu}=x^{\mu}+\varepsilon^{l} \Delta_{l}^{\mu}(x) \\
\varphi_{i}(x) \rightarrow \varphi_{i}^{\prime}\left(x^{\prime}\right)=\varphi_{i}(x)+\varepsilon^{l} \Lambda_{i, l}(\varphi, \partial \varphi)
\end{gathered}
$$

for a given set of functions $\Delta_{l}^{\mu}(x)$ and $\Lambda_{i, l}(\varphi, \partial \varphi)$. By definition, (2) and (3) represent a continuous symmetry transformation if they leave (1) invariant.

It is readily seen that (2) and (3) may be cast in the form of infinitesimal scale transformations via

$$
x^{\mu}+\varepsilon^{l} \Delta_{l}^{\mu}(x)=\lambda(x) x^{\mu}
$$




$$
\varphi_{i}(x)+\varepsilon^{l} \Lambda_{i, l}(\varphi, \partial \varphi)=\bar{\lambda}(x) \varphi_{i}(x)
$$

In what follows, $\lambda(x)$ and $\bar{\lambda}(x)$ are referred to as scaling operators of magnitude substantially close to one

$$
\lambda(x)=\bar{\lambda}(x)=1 \pm \varepsilon, \quad \varepsilon<<1
$$

In keeping with the standard terminology, the scaling operator is deemed local if it is coordinate-dependent and global otherwise. On account of (4), the infinitesimal Noether transformations are equivalent to local scaling operations that have no effect on the action. This insight suggests that invariance under properly defined scale transformations, and in particular self-similarity, must lie behind the fundamental symmetries of effective field theories.

\section{Self-similarity as underlying source of invariance}

Consider again the set of observables $q=\left\{q_{1}, q_{2}, \ldots, q_{N}\right\}$. Self-similarity in field theory and statistical physics enters through the generalized homogeneous function [3]

$$
f\left(\rho^{a} q\right)=f\left(\rho^{a} q_{1}, \rho^{a} q_{2}, \ldots, \rho^{a} q_{N}\right)=\rho f(q), \quad \rho>0
$$

Some textbooks display (6) in an alternative form, using the substitution

$$
\lambda=\rho^{a} \Rightarrow \rho=\lambda^{1 / a}
$$

which turns (6) into the condition

$$
f\left(q^{\prime}\right)=f(\lambda q)=\lambda^{1 / a} f(q)
$$


Covariance is a particular embodiment of self-similarity in the limit $a \rightarrow \infty$, in which case there is no distinction between the two sides of (8),

$$
f\left(q^{\prime}\right)=f(\lambda q)=f(q), \quad a \rightarrow \infty
$$

As detailed in [4-5], self-similarity has several applications in theoretical physics. Noteworthy examples include the behavior of classical oscillators, Newtonian gravitation, the virial theorem, Renormalization Group theory, random walk models, correlations and partition functions of Statistical Physics, and local scale invariance in field theory [6]. Equally important is the link between self-similarity and self-organized criticality (SOC), the latter being a universal paradigm for the onset of complex dynamics in systems outside equilibrium. On the same note, we mention here that surprising connections between SOC and several unsettled topics of contemporary theoretical physics have been brought up in $[7-11,16]$.

To bridge the gap between self-similarity and SOC, one starts from the generalized homogeneous function of two variables

$$
f\left(\lambda^{a} q_{1}, \lambda^{b} q_{2}\right)=\lambda f\left(q_{1}, q_{2}\right), \quad \lambda>0
$$

and show that (10) satisfies the power-law scaling [3]

$$
f\left(q_{1}, q_{2}\right)=\left|q_{1}\right|^{1 / a} \Phi\left(q_{2} /\left|q_{1}\right|^{b / a}\right)
$$

where $\Phi(\ldots)$ is called the scaling or cutoff function. (11) can be presented in the alternative form 


$$
\Phi\left(q_{2} /\left|q_{1}\right|^{b / a}\right)=f\left(1, q_{2} /\left|q_{1}\right|^{b / a}\right)
$$

A generic paradigm for SOC is the sandpile model, whose "avalanche"-size probability distribution is given by

$$
\begin{gathered}
P(s, L) \sim s^{-\tau_{s}} \Phi\left(s / s_{c}\right), \quad s>>1, L>>1 \\
s_{c}(L) \sim L^{D}, L>>1
\end{gathered}
$$

in which $s$ is the avalanche size, $L$ the system size and $\tau_{s}, D$ denote the avalanche-size exponent and its dimension, respectively. Term by term identification of (11) and (13)(14) yields

$$
a=b=-1 / \tau_{S}
$$

The key observation of this report is that the scaling operator $\lambda$ of (5) can be naturally interpreted as a phase angle. Appealing to the concept of analytic continuation in Euclidean field theory [12-13], leads to

$$
\lambda=\exp \theta=\exp \left(-i \theta_{E}\right)
$$

in which $\theta_{E}$ is the Euclidean phase. Since any phase angle is the product of an angular frequency $\omega$ with time $t=-i t_{E}$, we can write

$$
\theta=\omega t=\omega\left(-i t_{E}\right)=-i \omega t_{E}
$$


and the Euclidean scaling operator $\lambda_{E}$ can be presented as

$$
\theta_{E}=\omega t_{E} \Rightarrow \lambda_{E}=\exp \left(-i \theta_{E}\right)
$$

An elementary example showing the equivalence of infinitesimal scaling and rotation operations is discussed in the Appendix B.

\section{Self-similarity and the Lorentz group}

The Lorentz group represents the group of linear coordinate transformations

$$
x^{\mu} \rightarrow x^{\prime \mu}=\Lambda_{v}^{\mu} x^{\nu}
$$

that leave invariant the relativistic interval

$$
s^{2}=\eta_{\mu v} x^{\mu} x^{v}=t^{2}-\sum_{1}^{3} x_{i}^{2}
$$

Infinitesimal coordinate transformations are given by [2]

$$
\Lambda_{v}^{\mu}=\delta_{v}^{\mu}+\omega_{v}^{\mu}, \quad \omega_{\mu v}=-\omega_{v \mu}
$$

and a set of objects $\varphi^{i}(i=1,2, \ldots, n)$ transform as

$$
\varphi^{i} \rightarrow \Lambda_{j}^{i} \varphi^{j}
$$

where, by (16)-(18), the exponential matrix $\Lambda_{j}^{i}$ acts as scaling operator and takes the form

$$
\lambda \Rightarrow \Lambda_{j}^{i}=\left[\exp \left(-\frac{1}{2} \omega_{\mu v} J^{\mu v}\right)\right]_{j}^{i}
$$


Here, $\omega_{\mu v}$ denotes the six independent elements of the antisymmetric matrix entering (21) and $J^{\mu \nu}$ are the group generators. A similar line of reasoning is outlined in [14], where Lorentz transformations are regarded as similitudes.

\section{Self-similarity and gauge theory}

The QED Lagrangian written in the Feynman gauge is given by [2]

$$
L_{Q E D}=\bar{\psi}\left(i \gamma^{\mu} \partial_{\mu}-m\right) \psi-\frac{1}{4} F_{\mu \nu} F^{\mu \nu}-\frac{1}{2}\left(\partial_{\mu} A^{\mu}\right)^{2}-e A_{\mu} \bar{\psi} \gamma^{\mu} \psi
$$

Demanding that the QED Lagrangian conforms to local $U(1)$ gauge symmetry amounts to requiring that (24) stays unchanged under

$$
\psi(x) \rightarrow \exp [i e \theta(x)] \psi(x)
$$

and

$$
A_{\mu}(x) \rightarrow A_{\mu}(x)-\partial_{\mu} \theta(x)=\exp \left[\sigma(\theta(x)] A_{\mu}(x)\right.
$$

By (16)-(18), (25) and (26) can be interpreted as local scaling operations in field space defined by

$$
\begin{gathered}
\ln \lambda \Rightarrow \theta(x)=\omega_{\psi}(x)\left|t_{E}\right| \\
\ln \lambda \Rightarrow \sigma(\theta(x))=\omega_{A}(x)\left|t_{E}\right|
\end{gathered}
$$

It is known that imposing local gauge invariance leads to the introduction of the covariant derivative operator 


$$
D_{\mu} \psi=\left(\partial_{\mu}+i e A_{\mu}\right) \psi
$$

which transforms in the same manner as (25), that is, as a local scaling operation in field space

$$
D_{\mu} \psi \rightarrow \exp [i e \theta(x)] D_{\mu} \psi
$$

\section{Self-similarity and General Relativity}

The gauge field concept brought up in the last section may be built from a straightforward geometric interpretation [13]. Consider the parallel transport of a complex vector $|\psi\rangle$ round a closed rectangular loop. The difference between the value of $|\psi\rangle$ at the starting point $\left(|\psi\rangle_{0}\right)$ and at the end point $|\psi\rangle_{0} \rightarrow|\psi\rangle_{f}$ assumes the form

$$
\Delta \psi=\psi_{f}-\psi_{0}=-i g \Delta S^{\mu v} F_{\mu \nu} \psi
$$

in which $\Delta S^{\mu v}$ denotes the area enclosed by the rectangle and the strength of the gauge field is

$$
F_{\mu v}=\partial_{\mu} A_{v}-\partial_{v} A_{\mu}-i g\left[A_{\mu}, A_{v}\right]
$$

It is seen that the effect of parallel transport is to induce a non-vanishing rotation of $|\psi\rangle$ in internal space proportional to the strength of the gauge field. Likewise, the curvature tensor of General Relativity (GR) may be motivated through similar arguments. Taking a vector $V^{\kappa}$ on a round trip by parallel transport, the difference between the initial and final components of the vector amounts to 


$$
\Delta V^{\kappa}=\frac{1}{2} R_{\lambda \mu \nu}^{\kappa} V^{\lambda} \Delta S^{\mu \nu}
$$

This equation faithfully replicates (31) and signals the presence of a gravitational field via the curvature tensor $R_{\lambda \mu v}^{\kappa}$. The geometric analogy between gauge theory and GR is captured in the table below.

\begin{tabular}{|c|c|}
\hline Gauge Theory & General Relativity \\
\hline Gauge transformation & Coordinate transformation \\
\hline Gauge group & $\begin{array}{c}\text { Group of coordinate } \\
\text { transformations }\end{array}$ \\
\hline Gauge potential $A_{\mu}$ & Connection coefficient $\Gamma_{\mu \nu}^{\kappa}$ \\
\hline Field strength $F_{\mu \nu}$ & Curvature tensor $R_{\lambda \mu \nu}^{\kappa}$ \\
\hline
\end{tabular}

Comparison between the geometry of gauge and gravitational fields

In closing, we note that the explicit connection between the spacetime geometry of GR and self-similarity is detailed in $[15,17]$. It is worth recalling, however, that the onset of scale invariance in GR is not universal but context dependent. In general, coordinate invariance and scale invariance of the gravitational action are known to be fundamentally incompatible [18].

\section{APPENDIX A}

The group of affine transformations is a $n(n+1)$ - dimensional Lie group defined by

$$
\mathbf{q}^{\prime}=U \mathbf{q}+\mathbf{a}
$$


in which $U$ is a non-singular $(n \times n)$ matrix and $\mathbf{q}, \mathbf{q}^{\prime}$, and $\mathbf{a}$ are vectors. The case $\mathbf{a}=0$ represents an affine rotation, while $U=I$ corresponds to a translation. The transformation (A1) in which the matrix $U$ is the product of an orthogonal matrix by a real number $\lambda \neq \pm 1$ is called a similitude. Furthermore, if $U=\lambda I$, the similitude becomes a self-similar transformation (homothety) described by

$$
\mathbf{q}^{\prime}=\lambda I \mathbf{q}+\mathbf{a}
$$

It is apparent that a self-similar transformation is a composition of a dilation, a rotation and a translation of the types encoded in the Lorentz and gauge groups of effective field theory.

\section{$\underline{\text { APPENDIX B }}$}

The standard representation of the Dirac field as a doublet of left and right components is

$$
\psi(x)=\left[\begin{array}{l}
\psi_{R}(x) \\
\psi_{L}(x)
\end{array}\right]
$$

A local transformation $\psi(x) \rightarrow \psi^{\prime}(x)$ is formally identical to a $U(1)$ rotation in field space given by

$$
\psi^{\prime}(x)=\left[\begin{array}{l}
\psi_{R}^{\prime}(x) \\
\psi_{L}^{\prime}(x)
\end{array}\right]=\left[\begin{array}{cc}
\cos \theta(x) & \sin \theta(x) \\
-\sin \theta(x) & \cos \theta(x)
\end{array}\right]\left[\begin{array}{l}
\psi_{R}(x) \\
\psi_{L}(x)
\end{array}\right]
$$

For infinitesimal rotations defined by $\theta(x)<<1$, (B2) turns into 


$$
\left[\begin{array}{l}
\psi_{R}^{\prime}(x) \\
\psi_{L}^{\prime}(x)
\end{array}\right]=\left[\begin{array}{cc}
1 & \theta(x) \\
-\theta(x) & 1
\end{array}\right]\left[\begin{array}{l}
\psi_{R}(x) \\
\psi_{L}(x)
\end{array}\right]
$$

By (16), the angle $\theta(x)$ can be mapped to an infinitesimal scaling operation as in

$$
\lambda(x)=1+\theta(x) \approx \exp (\theta(x))=\exp \left[-i \theta_{E}(x)\right]
$$

\section{References}

1. Falconer, K. J., “The Geometry of Fractal Sets”, Cambridge Univ. Press, 1985.

2. Maggiore, M., "A Modern Introduction to Quantum Field Theory”, Oxford Univ. Press, 2005 .

3. Christensen, K. and Moloney, N. R., "Complexity and Criticality", Imperial College Press, 2005 .

4. Altenberger, A. R. and Dahler, J. S., "The role of self-similarity in Renormalization Group theory", in Advances in Chemical Physics, vol. 123, pp. 267-354, John Wiley \& Sons, 2002.

5. Landau L., Lifshitz E., “Mechanics, vol.1”, Butterworth-Heinemann, 1987.

6. https://www.sciencedirect.com/science/article/abs/pii/Sog60077904002942

7. Available at the following site:

https://www.researchgate.net/publication/342183452_Universal_Constants_from_Sel f-similarity and Adiabatic Invariance

8. Available at the following site:

https://www.researchgate.net/publication/341264527 Emergence of Lie Groups an d Gauge Symmetries from Complex Dynamics

9. Available at the following site: 
https://www.researchgate.net/publication/340819356_Emergence_of_Lagrangian_Fie ld Theory from Self-Organized Criticality

10. Available at the following site:

https://www.researchgate.net/publication/340595503 Quantum Field Theory as M anifestation of Self-Organized Criticality\#fullTextFileContent

11. Available at the following site:

https://www.researchgate.net/publication/340435561_Solving the Flatness_and_Ho rizon Problems via Self-Organized Criticality\#fullTextFileContent

12. Duncan A., "The Conceptual Framework of Quantum Field Theory", Oxford Univ. Press, 2012.

13. Ryder, L. H., "Quantum Field Theory”, Cambridge Univ. Press, 1989.

14. Rosenfeld, B. and Wiebe, B., "Geometry of Lie Groups", Springer, 1997.

15. https://projecteuclid.org/download/pdf 1/euclid.cmp/1103859930

16. Available at the following site:

https://www.researchgate.net/publication/341110733 Complex Dynamics as Found

ation of Relativistic Spacetime\#fullTextFileContent

17. https://cds.cern.ch/record/603700/files/0302004.pdf

18. https://arxiv.org/pdf/gr-qc/0312024.pdf 